\title{
The Nonlinear Hydroelastic Response of a Semi-Infinite Elastic Plate Floating on a Fluid due to Incident Progressive Waves
}

\author{
Ping Wang ${ }^{1,2}$ \\ ${ }^{1}$ School of Mathematics and Physics, Qingdao University of Science and Technology, 99 Songling Road, Qingdao 266061, China \\ ${ }^{2}$ Shanghai Institute of Applied Mathematics and Mechanics, Shanghai University, 149 Yanchang Road, Shanghai 200072, China \\ Correspondence should be addressed to Ping Wang; pingwang2003@126.com
}

Received 26 October 2014; Revised 25 February 2015; Accepted 4 March 2015

Academic Editor: Klaus Kirsten

Copyright (c) 2015 Ping Wang. This is an open access article distributed under the Creative Commons Attribution License, which permits unrestricted use, distribution, and reproduction in any medium, provided the original work is properly cited.

\begin{abstract}
The nonlinear hydroelastic response of very large floating structures (VLFSs) or an ice sheet floating on the surface of deep water, idealized as a semi-infinite thin elastic plate, is investigated analytically in the case of nonlinear incident waves. Assuming that the fluid is inviscid and incompressible and the motion is irrotational, we consider incident progressive waves with a given angular frequency within the framework of potential flow theory. With the aid of the homotopy analysis method (HAM), the convergent analytical series solutions are derived by solving the simultaneous equations in which we apply a convergence-control parameter to obtain convergent solutions with relatively few terms. The clear calculation results are represented to show nonlinear wave-plate interaction. The effects of different physical parameters, including incident wave amplitude, Young's modulus, the thickness and density of the plate on the wave scattering, and the hydroelastic response of the floating plate, are considered. We find that the variations of the plate stiffness, thickness, and density greatly change amount of wave energy which is reflected into the open water region and is transmitted into the plate-covered region. Further, the hydroelastic response of the plate also can be affected by the amplitude of incident wave.
\end{abstract}

\section{Introduction}

In recent decades, there have been intensive researches on hydroelastic interaction between water waves and elastic plates in the area of ocean engineering and polar engineering in view of their academic research and applications. The very large floating structures (VLFSs), which are expected to serve as floating airports, artificial floating islands, ultralarge ships, or even mobile offshore bases, are mathematically assumed as elastic plates floating on a fluid for their strong flexibility and huge horizontal scales compared to vertical scales and characteristic wavelength. Accordingly, the hydroelastic deformation in the response of the structure to water waves is a predominant factor to design and maintain a safe and economic VLFS. Early studies in this field were mainly focused on hydroelastic interaction between the water waves and the large ice sheets which were also idealized as floating elastic plates [1], and the elastic plates are usually assumed to be semi-infinitely long in comparison with the wavelength of the incident wave $[2,3]$. A comprehensive summary of the early work can be found in some review papers such as [2-5]. Unfortunately, mathematical methods on solving nonlinear problems are not well developed, so most of the present analytical researches on the hydroelastic interaction between water waves and an elastic plate still are in the scope of linear theory, which only can describe small-amplitude waves. Taking a semi-infinite elastic plate by using the eigenfunction expansion method, for example, Fox and Squire [4] investigated the reflection and transmission of ocean waves at the margin of an ice sheet which is idealized as a semi-infinite elastic plate and then determined the expansion coefficients via matching the velocity potential and the pressure at the interface of the open water region and the plate-covered region and enforcing the zero shear and bending moment at the free end. However, Fox and Squire [4] also stated that the beat Lagrange's multiplier is very difficult to choose and the mathematical calculations become impossible for the unwise choice of the multipliers. Fox and Squire [3] subsequently improved their original method by removing 
the free end boundary conditions from the error function and then reducing the number of the Lagrange's multiplier from three to one and analyzed the hydroelastic response of a semi-infinite elastic with a free edge due to the obliquely incident waves. Following the framework of Fox and Squire [3], Sahoo et al. [6] developed a new inner product based on the Fourier analysis within which the original eigenfunctions for the plate-covered region were orthogonal. It is observed that the hydrodynamic behaviors of the floating semi-infinite elastic plate with different edge conditions, including a free edge, a simply supported edge, and built-in edge condition, depend on the wave conditions, the geometrical settings, and the edge conditions. Meanwhile, Teng et al. [7] optimized Fox and Squire's method [3] by removing the Lagrange multipliers from the error function completely and improved the method of Sahoo et al. [6] through employing the eigenfunctions in the open water region and the plate-covered region, respectively. It is demonstrated with examples that the solver for the associated linear system is simplified. In $\mathrm{Xu}$ and $\mathrm{Lu}$ [8], the eigenfunction expansion method was optimized by using the orthogonality property of eigenfunctions in the open water region. And numerical analysis showed that this modified method was effective and had higher convergence than the previous results in Sahoo et al. [6] and Teng et al. [7]. With the aid of the methods of matched eigenfunction expansion and the inner product of the two-layer fluid, Lin and $\mathrm{Lu}$ [9] extended the study of Fox and Squire [3] to the case that a semi-infinite elastic plate floating on a two-layer fluid of finite depth is subjected to the obliquely incident waves and found a critical angle for the incident waves of the surface wave mode and three critical angles for the incident waves of the interfacial wave mode, which are related to the existence of the propagating waves.

More importantly, it is inevitable that large-amplitude waves will occur more often in the future with the growing frequency of extreme weather events on a warmer and warmer Earth. Thus, some scholars investigated nonlinear hydroelastic interaction between semi-infinite elastic plates and water waves by using the well-known perturbation method. Forbes [10] studied the nonlinear interaction between two-dimensional periodic waves with a constant speed and an elastic ice sheet floating on a fluid of infinite depth by using the perturbation expansion in the half-wave height and found that approximate solutions for the periodic waves had certain features in common with capillary-gravity waves. Forbes [11] subsequently improved their original perturbation method by introducing Newton-Raphson techniques to approximate the Fourier coefficients and confirmed the existence of multiple solutions for the nonlinear equations describing the shape of nonlinear periodic waves, and extremely large-amplitude waves were also found to exist. With the perturbation method in a similar way, VandenBroeck and Părău [12] extended the study of Forbes [11] to the case of large-amplitude periodic waves and unfortunately found that the perturbation method cannot give any information for very steep waves because this method has to depend on small physical parameters. Further, Milewski et al. [13] applied the asymptotic and numerical methods to analyze the hydroelastic solitary waves propagating under

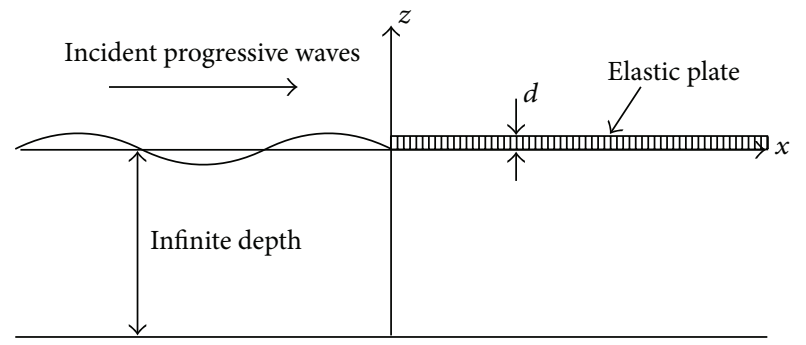

FIGURE 1: Schematic diagram for the interaction between incident progressive waves and a semi-infinite elastic plate floating on a fluid of infinite depth.

a semi-infinite elastic plate. It is found that wave packet solitary waves bifurcate from nonlinear periodic waves of minimum speed for the unforced problem, and when the problem is forced by a moving load, steady responses are possible at all subcritical speeds for small-amplitude forcing, while there was a transcritical range of forcing speeds for which there are no steady solutions for larger loads. Finally, it is also noted that these elastic models do not have a clear conservation form for the elastic potential energy.

It is well known that the traditional perturbation and asymptotic techniques depend on the small physical parameters and approximations of nonlinear problems usually break down when the nonlinearity becomes strong. Therefore, they are only valid for weakly nonlinear problems. In this paper, we apply the homotopy analysis method (HAM) developed by Liao [14], a powerful analytic method for highly nonlinear problems, to consider the nonlinear hydroelastic response of a semi-infinite elastic plate floating on a fluid due to incident progressive waves. Further, we investigate the dynamic influences of some important physical parameters, including incident wave amplitude, Young's modulus, and the density and the thickness of the plate, on nonlinear hydroelastic response of the floating plate and the wave scattering.

\section{Mathematical Formulation}

We consider the nonlinear hydroelastic interaction between incident progressive waves and a semi-infinite elastic plate floating on infinitely deep water for the two-dimensional case, as shown in Figure 1. Cartesian coordinates $o x z$ are chosen such that $z=0$ represents the undisturbed water surface. The $x$-axis points horizontally rightward and the $z$ axis points vertically upward. The semi-infinite elastic plate, which floating on a fluid, expends rightward from $x=0$ to infinity along the $x$-axis without draft. Then the whole fluid domain is divided into two regions: an open water region $(-\infty<x<0)$ and a plate-covered region $(0<x<+\infty)$. Under the assumptions that the fluid is inviscid and incompressible and the motion is irrotational, the velocity potential $\phi(x, z, t)$ satisfies the Laplace equation based on the potential flow theory:

$$
\frac{\partial^{2} \phi}{\partial x^{2}}+\frac{\partial^{2} \phi}{\partial z^{2}}=0, \quad(z \leq \zeta(x, t),-\infty<x<+\infty) .
$$


We consider the nonlinear hydroelastic interaction between incident progressive waves and a semi-infinite elastic plate floating on infinitely deep water for the twodimensional case, as shown in Figure 1. Cartesian coordinates $o x z$ are chosen such that $z=0$ represents the undisturbed water surface. The $x$-axis points horizontally rightward and the $z$-axis points vertically upward. The semi-infinite elastic plate floating on a fluid expends rightward from $x=0$ to infinity along the $x$-axis without draft. Then the whole fluid domain is divided into two regions: an open water region $(-\infty<x<0)$ and a plate-covered region $(0<x<$ $+\infty)$. Under the assumptions that the fluid is inviscid and incompressible and the motion is irrotational, the velocity potential $\phi(x, z, t)$ satisfies the Laplace equation based on the potential flow theory:

$$
\frac{\partial^{2} \phi}{\partial x^{2}}+\frac{\partial^{2} \phi}{\partial z^{2}}=0, \quad(z \leq \zeta(x, t),-\infty<x<+\infty),
$$

where $\zeta(x, t)$ is the wave surface elevation. The bottom boundary condition at deep flat rigid seabed reads

$$
\frac{\partial \phi}{\partial z}=0, \quad(z=-\infty,-\infty<x<+\infty) .
$$

The nonlinear kinematic boundary condition on the unknown surface can be written as

$$
\frac{\partial \zeta}{\partial t}+\frac{\partial \phi}{\partial x} \frac{\partial \zeta}{\partial x}-\frac{\partial \phi}{\partial z}=0, \quad(z=\zeta(x, t),-\infty<x<+\infty) .
$$

In the open water region, the nonlinear dynamic boundary condition on the unknown surface reads

$$
\frac{\partial \phi}{\partial t}+\frac{1}{2}|\nabla \phi|^{2}+g \zeta=0, \quad(z=\zeta(x, t),-\infty<x<0),
$$

where $g$ is the gravitational acceleration. Combining (4) and (5) gives the boundary condition

$$
\begin{array}{r}
\frac{\partial^{2} \phi}{\partial t^{2}}+g \frac{\partial \phi}{\partial z}+\frac{\partial f}{\partial t}-g \frac{\partial \phi}{\partial x} \frac{\partial \zeta}{\partial x}=0, \\
(z=\zeta(x, t),-\infty<x<0),
\end{array}
$$

where

$$
f=\frac{1}{2}\left[\left(\frac{\partial \phi}{\partial x}\right)^{2}+\left(\frac{\partial \phi}{\partial z}\right)^{2}\right] .
$$

In the plate-covered region, under the assumption that any fluid particle which is once between the thin elastic plate and the water surface will remain on it, the dynamic boundary condition on the fluid-plate interface can be written as

$$
\begin{array}{r}
\frac{\partial \phi}{\partial t}+\frac{1}{2}|\nabla \phi|^{2}+g \zeta+\frac{1}{\rho}\left[D \frac{\partial^{4} \zeta}{\partial x^{4}}+m_{\mathrm{e}}\left(\frac{\partial^{2} \zeta}{\partial t^{2}}+g\right)\right]=0, \\
(z=\zeta(x, t), 0<x<+\infty),
\end{array}
$$

where $\rho$ is the fluid density. For a thin homogeneous elastic plate with uniform mass density $\rho_{\mathrm{e}}$ and constant thickness $d$, $m_{\mathrm{e}}=\rho_{\mathrm{e}} d$ is the mass per unit area of the plate. $D$ is the flexural rigidity of the plate which is expressed as $D=E d^{3} /[12(1-$ $\left.v^{2}\right)$ ] by Young's modulus $E$, Poisson's ratio $v$, and the thickness $d$ of the plate. We partially combine (4) and (8) to gain the boundary conditions for the plate-covered region as follows:

$$
\begin{array}{r}
\frac{\partial^{2} \phi}{\partial t^{2}}+g \frac{\partial \phi}{\partial z}+\frac{\partial f}{\partial t}-g \frac{\partial \phi}{\partial x} \frac{\partial \zeta}{\partial x}+\frac{1}{\rho}\left(D \frac{\partial^{5} \zeta}{\partial t \partial x^{4}}+m_{\mathrm{e}} \frac{\partial^{3} \zeta}{\partial t^{3}}\right)=0, \\
(z=\zeta(x, t),-\infty<x<0) .
\end{array}
$$

Further, considering the continuities of the pressure and the velocity on the boundary between the plate-covered region and the open water region, we express the matching conditions as

$$
\begin{array}{cl}
\phi(0-, z, t) & =\phi(0+, z, t), \quad-\infty<z<\zeta(x, t), \\
\frac{\partial \phi(0-, z, t)}{\partial x} & =\frac{\partial \phi(0+, z, t)}{\partial x}, \quad-\infty<z<\zeta(x, t) .
\end{array}
$$

For the elastic plate freely floating on the water surface, it is reasonable that the motion of the plate satisfies the free edge conditions as follows:

$$
\begin{aligned}
& \frac{\partial^{2} \zeta(x, t)}{\partial x^{2}}=0, \quad x=0, z=\zeta(x, t), \\
& \frac{\partial^{3} \zeta(x, t)}{\partial x^{3}}=0, \quad x=0, z=\zeta(x, t),
\end{aligned}
$$

where $t$ is a given time. In this paper, we choose $t=\pi$ for matching conditions (10) and the free edge conditions (11). Now the unknown velocity potential $\phi(x, z, t)$ and the plate deflection $\zeta(x, t)$ for two different water regions are governed by (2)-(4), (6), (9), (10), and (11). For the given angular frequency $\omega$, the convergent analytical series solutions for $\phi(x, z, t)$ and $\zeta(x, t)$ will be obtained based on the HAM in the subsequent section.

\section{Analytical Approach Based on the HAM}

The homotopy analysis method (HAM) is an analysis approximation method developed by Liao [14] based on the concept of homotopy in algebraic topology. Different from the traditional perturbation method, the HAM is completely independent of any small or large physical parameters. Thus it is valid to highly nonlinear problems without any small or large parameter. More importantly, the HAM provides us with a convenient way to control and adjust the convergence of the approximate series solutions by means of introducing convergence-control parameters. Further, the HAM gives us great freedom in the choice of the initial guess, the basis solution functions, and the equation type of linear subproblems in order to simplify the solution procedure. Recently, the HAM has been successfully applied to solve many highly nonlinear problems in many fields. In hydrodynamics, for example, Liao [15] utilized the homotopy-based multiple-variable method to analyze the nonlinear interactions of periodic traveling 
waves in deep water. $\mathrm{Xu}$ et al. [16] further extended the study of Liao [15] to the case of steady-state system for the nonlinear interaction of two trains of waves propagating in water of definite depth by using the HAM in a similar way and found six different steady-state resonant waves. It is noted that Wang and $\mathrm{Lu}$ [17] employed the HAM to consider nonlinear hydroelastic progressive waves traveling in an elastic plate in deep water. And graphical representations of the series solutions show that several physical parameters have important influences on the hydroelastic response of the elastic plate floating on the surface of deep water. For details on the HAM, please refer to the two books by Liao $[18,19]$.

3.1. Continuous Variations. Let $q \in[0,1]$ denote the embedding parameter and let $\phi_{0}(x, z, t)$ and $\zeta_{0}(x, t)$ denote the initial approximations of the velocity potential $\phi(x, z, t)$ and wave elevation $\zeta(x, t)$, respectively. Instead of solving these nonlinear equations (2)-(4), (6), (9), (10), and (11) directly, in the frame of the HAM we construct two continuous variations $\Phi(x, z, t ; q)$ and $\eta(x, t ; q)$ which are called homotopies in topology, such that as $q$ increases from 0 to 1 , $\Phi(x, z, t ; q)$ deforms continuously from its initial approximation $\phi_{0}(x, z, t)$ to the exact solution $\phi(x, z, t)$ of the original problem, so does $\eta(x, t ; q)$ from the approximation $\zeta_{0}(x, t)$ to $\zeta(x, t)$. Those two continuous variations are governed by a new family of nonlinear partial differential equations, namely, the so-called zeroth-order deformation equations:

$$
\begin{gathered}
\frac{\partial^{2} \Phi(x, z, t ; q)}{\partial x^{2}}+\frac{\partial^{2} \Phi(x, z, t ; q)}{\partial z^{2}}=0 \\
(z \leq \eta(x, t ; q),-\infty<x<+\infty),
\end{gathered}
$$

subject to the bottom condition

$$
\frac{\partial \Phi(x, z, t ; q)}{\partial z}=0, \quad(z=-\infty,-\infty<x<+\infty) .
$$

In the open water region, the two boundary conditions on the unknown free surface $z=\eta(x, t ; q)(-\infty<x<0)$

$$
\begin{aligned}
(1-q) & \mathscr{L}_{1}^{L}\left[\Phi(x, z, t ; q)-\phi_{0}(x, z, t)\right] \\
= & q c_{0} \mathcal{N}_{1}^{L}[\Phi(x, z, t ; q), \eta(x, t ; q)], \\
(1-q) & {\left[\eta(x, t ; q)-\zeta_{0}(x, t)\right] } \\
= & q c_{0} \mathcal{N}_{2}^{L}[\eta(x, t ; q), \Phi(x, z, t ; q)],
\end{aligned}
$$

where $c_{0}$ denotes a nonzero convergence-control parameter and the superscript $L$ identifies the open water region. $\mathscr{L}_{1}^{L}$ is an auxiliary linear operator with the property $\mathscr{L}_{1}^{L}[0]=0 . \mathcal{N}_{1}^{L}$ and $\mathscr{N}_{2}^{L}$ are nonlinear differential operators defined by

$$
\begin{aligned}
\mathcal{N}_{1}^{L}[\Phi(x, z, t ; q), \eta(x, t ; q)] \\
=\frac{\partial^{2} \Phi(x, z, t ; q)}{\partial t^{2}}+g \frac{\partial \Phi(x, z, t ; q)}{\partial z}+\frac{\partial F(x, z, t ; q)}{\partial t} \\
\quad-g \frac{\partial \Phi(x, z, t ; q)}{\partial x} \frac{\partial \eta(x, t ; q)}{\partial x}, \\
\mathcal{N}_{2}^{L}[\eta(x, t ; q), \Phi(x, z, t ; q)] \\
=g \eta(x, t ; q)+F(x, z, t ; q)+\frac{\partial \Phi(x, z, t ; q)}{\partial t},
\end{aligned}
$$

with the definition

$$
F(x, z, t ; q)=\frac{1}{2}\left[\left(\frac{\partial \Phi(x, z, t ; q)}{\partial x}\right)^{2}+\left(\frac{\partial \Phi(x, z, t ; q)}{\partial z}\right)^{2}\right] .
$$

Similarly, in the plate-covered region, the so-called zeroth-order deformation equations are the governing equation (12) which is subject to the bottom condition (13) and the following two boundary conditions on the unknown platecovered surface $z=\eta(x, t ; q)(0<x<+\infty)$ :

$$
\begin{aligned}
(1-q) & \mathscr{L}_{1}^{R}\left[\Phi(x, z, t ; q)-\phi_{0}(x, z, t)\right] \\
= & q \mathcal{c}_{0} \mathscr{N}_{1}^{R}[\Phi(x, z, t ; q), \eta(x, t ; q)], \\
(1-q) & \mathscr{L}_{2}^{R}\left[\eta(x, t ; q)-\zeta_{0}(x, t)\right] \\
= & q \mathcal{c}_{0} \mathcal{N}_{2}^{R}[\eta(x, t ; q), \Phi(x, z, t ; q)],
\end{aligned}
$$

where the superscript $R$ identifies the plate-covered region. $\mathscr{L}_{1}^{R}$ and $\mathscr{L}_{2}^{R}$ are auxiliary linear operators with $\mathscr{L}_{i}^{R}[0]=0$, $(i=1,2) . \mathscr{N}_{1}^{R}$ and $\mathcal{N}_{2}^{R}$ are nonlinear differential operators defined by

$$
\begin{aligned}
\mathcal{N}_{1}^{R}[\Phi(x, z, t ; q), \eta(x, t ; q)] \\
=\frac{\partial^{2} \Phi(x, z, t ; q)}{\partial t^{2}}+g \frac{\partial \Phi(x, z, t ; q)}{\partial z} \\
\quad+\frac{\partial F(x, z, t ; q)}{\partial t}-g \frac{\partial \Phi(x, z, t ; q)}{\partial x} \frac{\partial \eta(x, t ; q)}{\partial x} \\
\quad+\frac{1}{\rho}\left[D \frac{\partial^{5} \eta(x, t ; q)}{\partial x^{4} \partial t}+m_{\mathrm{e}} \frac{\partial^{3} \eta(x, t ; q)}{\partial t^{3}}\right] . \\
\mathcal{N}_{2}^{R}[\eta(x, t ; q), \Phi(x, z, t ; q)] \\
=g \eta(x, t ; q) \\
\quad+\frac{1}{\rho}\left[D \frac{\partial^{4} \eta(x, t ; q)}{\partial x^{4}}+m_{\mathrm{e}}\left(\frac{\partial^{2} \eta(x, t ; q)}{\partial t^{2}}+g\right)\right] \\
\quad+\frac{\partial \Phi(x, z, t ; q)}{\partial t}+F(x, z, t ; q) .
\end{aligned}
$$


Note that the definitions for $\mathcal{N}_{1}^{L}[\cdot], \mathcal{N}_{2}^{L}[\cdot], \mathcal{N}_{1}^{R}[\cdot]$, and $\mathcal{N}_{2}^{R}[\cdot]$ are based on the four nonlinear boundary conditions (6) and (5) and (9) and (8), respectively. It should be emphasized that the velocity potential $\Phi(X, z ; q)$ and wave elevation $\eta(X ; q)$ are defined as follows:

$$
\begin{gathered}
\Phi(x, z, t ; q)= \begin{cases}\Phi^{L}(x, z, t ; q), & -\infty<x<0, \\
\Phi^{R}(x, z, t ; q), & 0<x<+\infty,\end{cases} \\
\eta(x, t ; q)= \begin{cases}\eta^{L}(x, t ; q), & -\infty<x<0, \\
\eta^{R}(x, t ; q), & 0<x<+\infty .\end{cases}
\end{gathered}
$$

And their initial approximations are defined by

$$
\begin{gathered}
\phi_{0}(x, z, t)= \begin{cases}\phi_{0}^{L}(x, z, t), & -\infty<x<0, \\
\phi_{0}^{R}(x, z, t), & 0<x<+\infty,\end{cases} \\
\zeta_{0}(x, t)= \begin{cases}\zeta_{0}^{L}(x, t), & -\infty<x<0, \\
\zeta_{0}^{R}(x, t), & 0<x<+\infty .\end{cases}
\end{gathered}
$$

In addition, the matching conditions of the velocity and pressure at the boundary between the plate-covered region and the open water region are deformed into

$$
\begin{array}{r}
\Phi(0-, z, t ; q)=\Phi(0+, z, t ; q), \\
-\infty<z \leq \eta(x, t ; q), \\
\frac{\partial \Phi(0-, z, t ; q)}{\partial x}=\frac{\partial \Phi(0+, z, t ; q)}{\partial x}, \\
-\infty<z \leq \eta(x, t ; q) .
\end{array}
$$

And the free edge conditions are

$$
\begin{array}{ll}
\frac{\partial^{2} \eta(x, t ; q)}{\partial x^{2}}=0, & x=0, \\
\frac{\partial^{3} \eta(x, t ; q)}{\partial x^{3}}=0, & x=0 .
\end{array}
$$

In order to obtain the series solutions for the velocity potential $\phi(x, z, t)$ and wave elevation $\zeta(x, t)$, by Taylor's theorem, we expand $\Phi(x, z, t ; q)$ and $\eta(x, t ; q)$ into the Taylor series about the embedding parameter $q$ at $q=0$ as

$$
\begin{gathered}
\Phi(x, z, t ; q)=\phi_{0}(x, z, t)+\sum_{m=1}^{+\infty} \phi_{m}(x, z, t) q^{m} \\
\eta(x, t ; q)=\zeta_{0}(x, t)+\sum_{m=1}^{+\infty} \zeta_{m}(x, t) q^{m}
\end{gathered}
$$

where

$$
\begin{aligned}
& \left\{\phi_{m}(x, z, t), \zeta_{m}(x, t)\right\} \\
& \quad=\left.\frac{1}{m !} \frac{\partial^{m}}{\partial q^{m}}\{\Phi(\mathrm{x}, z, t ; q), \eta(x, t ; q)\}\right|_{q=0} .
\end{aligned}
$$

Assuming that $c_{0}$ could be so properly chosen that the above two series in (24) converge at $q=1$, then we have the so-called homotopy-series solutions as

$$
\begin{gathered}
\phi(x, z, t)=\Phi(x, z, t ; 1)=\phi_{0}(x, z, t)+\sum_{m=1}^{+\infty} \phi_{m}(x, z, t), \\
\zeta(x, t)=\eta(x, t ; 1)=\zeta_{0}(x, t)+\sum_{m=1}^{+\infty} \zeta_{m}(x, t),
\end{gathered}
$$

with the definitions

$$
\begin{gathered}
\phi_{m}(x, z, t)= \begin{cases}\phi_{m}^{L}(x, z, t), & -\infty<x<0, \\
\phi_{m}^{R}(x, z, t), & 0<x<+\infty,\end{cases} \\
\zeta_{m}(x, t)= \begin{cases}\zeta_{m}^{L}(x, t), & -\infty<x<0, \\
\zeta_{m}^{R}(x, t), & 0<x<+\infty .\end{cases}
\end{gathered}
$$

As shown later in Section 3.3, the unknown terms $\phi_{m}(x, z, t)$ and $\zeta_{m}(x, t)$ can be obtained by solving linear PDEs (38)-(41) for the open water region, (38), (39), (42), and (43) for the plate-covered region, and (44)-(45) at the boundary.

3.2. Solution Expressions. Considering the progressive gravity wave propagating periodically in $x$ with a given angular frequency $\omega$, the progressive waves can be expressed as the combinations of the wave traveling to the right and the wave traveling to the left in the open water region. From viewpoints of the physical background, we represent the progressive wave elevation in the open water region as follows:

$$
\zeta^{L}(x, t)=\sum_{i=0}^{+\infty}\left\{a_{1 i} \cos [i(k x-\omega t)]+a_{2 i} \cos [i(k x+\omega t)]\right\},
$$

where $a_{1 i}$ and $a_{2 i}$ are unknown coefficients to be determined. $k$ is the wave number for the open water region which satisfies [16] $\omega / \sqrt{g k}=\epsilon$, where the value of $\epsilon$ is not equal to 1 . Since the dispersion relation $\omega=\sqrt{g k}$ is valid only for the linear waves, in this paper we consider the case of $\epsilon=1.05$.

According to the linear wave theory, the governing equation (12) and the bottom condition (13), in the open water region the potential function $\phi(x, z, t)$, should be in the form

$$
\begin{aligned}
\phi^{L} & (x, z, t) \\
& =\sum_{i=0}^{+\infty}\left\{b_{1 i} \sin [i(k x-\omega t)]+b_{2 i} \sin [i(k x+\omega t)]\right\} \exp (i k z),
\end{aligned}
$$

where $b_{1 i}$ and $b_{2 i}$ are unknown coefficients to be determined.

In the plate-covered region, if there is no gap between the bottom surface of the thin elastic plate and the top surface of the fluid layer, it is reasonable that the plate or the surface fluid still travels periodically in the $k x-\omega t$. Then the plate 
deflection $\zeta(x, t)$ is mathematically represented as the form with (28):

$$
\zeta^{R}(x, t)=\sum_{i=0}^{+\infty} A_{i} \cos [i(k x-\omega t)]
$$

where $A_{i}$ is an unknown coefficient. $k$ is the wave number for the plate-covered water region which satisfies $\omega / \sigma=\epsilon[16]$, where the linear dispersion relations[8]:

$$
\sigma^{2}=\frac{\left(1+D k^{4} / \rho g\right) g k}{1+m_{\mathrm{e}} k / \rho} .
$$

With loss of generality, we consider the case of $\epsilon=1.05$ too.

Subsequently, we consider the expression of the potential function as

$$
\phi^{R}(x, z, t)=\sum_{i=0}^{+\infty} B_{i} \sin [i(k x-\omega t)] \exp (i k z),
$$

where $B_{i}$ is an unknown coefficient to be determined. Note that the potential function $\phi^{R}(x, z, t)$ defined by (32) also automatically satisfies the governing equation (12) and the bottom condition (13).

Equations (28) and (29) are called the solution expressions of $\zeta(x, t)$ and $\phi(x, z, t)$ in the open water region, and in the plate-covered region (30) and (32) are the solution expressions, respectively, which have important roles in the frame of the HAM.

As mentioned by Liao [19], according to the solution expressions (29) and (32), we have extremely large freedom to choose the initial approximations and the auxiliary linear operator which greatly simplify the process of solving nonlinear problems. Then in the two regions we construct the initial approximations of the potential function

$$
\begin{array}{r}
\phi_{0}^{L}(x, z, t)=\left[b_{0,1} \sin (k x-\omega t)+b_{0,2} \sin (k x+\omega t)\right] \exp (k z), \\
(-\infty<x<0),
\end{array}
$$

$$
\phi_{0}^{R}(x, z, t)=B_{0} \sin (k x-\omega t) \exp (k z), \quad(0<x<+\infty),
$$

respectively, where $b_{0,1}, b_{0,2}$, and $B_{0}$ are unknown coefficients. And we choose

$$
\begin{gathered}
\zeta_{0}^{L}(x, t)=a_{0,1} \cos (k x-\omega t)+a_{0,2} \cos (k x+\omega t), \\
\zeta_{0}^{R}(x, t)=A_{0} \cos (k x-\omega t)
\end{gathered}
$$

as the initial guess of $\zeta(x, t)$ for the open water region and the plate-covered region, respectively, where $a_{0,1}$ is a given amplitude of the initial incident progressive waves. $a_{0,2}$ and $A_{0}$ are unknown coefficients.

It should be noted that the HAM can provide extremely large freedom in the choice of auxiliary linear operators; then for simplifying the computation procedure we can only choose linear operators of $\Phi(x, z, t ; q)$ in $\mathcal{N}_{1}^{L}$ and $\mathcal{N}_{1}^{R}$ as the auxiliary linear operators $\mathscr{L}_{1}^{L}$ and $\mathscr{L}_{1}^{R}$ by means of the solution expressions (29) and (32) as follows:

$$
\begin{aligned}
\mathscr{L}_{1}^{L}[\Phi(x, z, t ; q)] & =\mathscr{L}_{1}^{R}[\Phi(x, z, t ; q)] \\
& =\frac{\partial^{2} \Phi(x, z, t ; q)}{\partial t^{2}}+g \frac{\partial \Phi(x, z, t ; q)}{\partial z} .
\end{aligned}
$$

And we choose linear operators of $\eta(x, t ; q)$ in $\mathcal{N}_{2}^{R}$ as the auxiliary linear operators by means of the solution expression (30) as

$$
\mathscr{L}_{2}^{R}[\eta(x, t ; q)]=\frac{\partial^{4} \eta(x, t ; q)}{\partial x^{4}}+\frac{\partial^{2} \eta(x, t ; q)}{\partial t^{2}}+\eta(x, t ; q) .
$$

3.3. High-Order Deformation Equations. The linear PDEs for the unknown $\phi_{m}(x, z, t)$ and $\zeta_{m}(x, t)$ can be derived directly from the zeroth-order deformation equations. Namely, differentiating the zero-order deformation equations (12)-(15) for the open water region, (12), (13), and (18) for the plate-covered region, and (22) and (23) at the boundary $m$ times with regard to $q$, then dividing them by $m$ ! and setting $q=0$, we have the so-called $m$ th-order deformation equations:

$$
\frac{\partial^{2} \phi_{m}}{\partial x^{2}}+\frac{\partial^{2} \phi_{m}}{\partial z^{2}}=0, \quad(z \leq 0,-\infty<x<+\infty),
$$

subject to the bottom condition

$$
\frac{\partial \phi_{m}}{\partial z}=0, \quad(z=-\infty,-\infty<x<+\infty),
$$

and in the open water region, the two boundary conditions at $(z=0,-\infty<x<0)$

$$
\begin{gathered}
\left.\mathscr{L}_{1}^{L}\left(\phi_{m}\right)\right|_{z=0}=c_{0} \Delta_{m-1}^{L, \phi}+H_{m} S_{m-1}^{L}-\bar{S}_{m}^{L}, \\
\zeta_{m}=c_{0} \Delta_{m-1}^{L, \zeta}+H_{m} \zeta_{m-1} .
\end{gathered}
$$

In the plate-covered region, the governing equation (38) which is subject to the bottom condition (39) and the following two boundary conditions at $(z=0,0<x<+\infty)$

$$
\begin{gathered}
\left.\mathscr{L}_{1}^{R}\left(\phi_{m}\right)\right|_{z=0}=c_{0} \Delta_{m-1}^{R, \phi}+H_{m} S_{m-1}^{R}-\bar{S}_{m}^{R}, \\
\mathscr{L}_{2}^{R}\left(\zeta_{m}\right)=c_{0} \Delta_{m-1}^{R, \zeta}+H_{m}\left(\frac{\partial^{4} \zeta_{m-1}}{\partial x^{4}}+\frac{\partial^{2} \zeta_{m-1}}{\partial t^{2}}+\zeta_{m-1}\right),
\end{gathered}
$$

where $H_{m}=H(m-2)$ and $H(\cdot)$ is the Heaviside step function.

The elaborated expressions for $\Delta_{m-1}^{L, \phi}, S_{m-1}^{L}, \bar{S}_{m}^{L}, \Delta_{m-1}^{L, \zeta}$ and $\Delta_{m-1}^{R, \phi}, S_{m-1}^{R}, \bar{S}_{m}^{R}, \Delta_{m-1}^{R, \zeta}$ are given in the Appendix.

At the boundary between the plate-covered region and the open water region, the matching conditions of the velocity and pressure of the free edge conditions are

$$
\begin{array}{cl}
\phi_{m}(0-, z, t) & =\phi_{m}(0+, z, t), \quad-\infty<z \leq 0, \\
\frac{\partial \phi_{m}(0-, z, t)}{\partial x}=\frac{\partial \phi_{m}(0+, z, t)}{\partial x}, & -\infty<z \leq 0 .
\end{array}
$$


And the free edge conditions are

$$
\begin{array}{ll}
\frac{\partial^{2} \zeta_{m}(x, t)}{\partial x^{2}}=0, & x=0, \\
\frac{\partial^{3} \zeta_{m}(x, t)}{\partial x^{3}}=0, & x=0 .
\end{array}
$$

Now we see that the unknown terms $\phi_{m}(x, z, t)$ and $\zeta_{m}(x, t)$ are governed by linear PDEs (38)-(41) for the open water region, (38), (39), (42), and (43) and (44) and (45) at the boundary. More importantly, these subproblems for $\phi_{m}$ and $\zeta_{m}$ are not only linear but also decoupled and then can easily be solved.

3.4. Optimal Convergence-Control Parameter. If we fix all model physical parameters in our nonlinear boundary-value problem, there will still be an unknown convergence-control parameter $c_{0}$ which is used to guarantee the convergence of the series solutions. The optimal value of $c_{0}$ corresponds to the fastest decrease of the total squared residuals of our nonlinear problem. The total squared residual $\varepsilon_{m}^{T}$ is defined by

$$
\varepsilon_{m}^{T}=\varepsilon_{m}^{L, \phi}+\varepsilon_{m}^{L, \zeta}+\varepsilon_{m}^{R, \phi}+\varepsilon_{m}^{R, \zeta},
$$

with the definitions

$$
\begin{aligned}
& \varepsilon_{m}^{L, \phi}=\frac{1}{1+M} \sum_{i=0}^{M}\left[\sum_{n=0}^{m} \Delta_{n}^{L, \phi}(i k \Delta x)\right]^{2}, \\
& \varepsilon_{m}^{R, \phi}=\frac{1}{1+M} \sum_{i=0}^{M}\left[\sum_{n=0}^{m} \Delta_{n}^{R, \phi}(i k \Delta x)\right]^{2}, \\
& \varepsilon_{m}^{L, \zeta}=\frac{1}{1+M} \sum_{i=0}^{M}\left[\sum_{n=0}^{m} \Delta_{n}^{L, \zeta}(i k \Delta x)\right]^{2}, \\
& \varepsilon_{m}^{R, \zeta}=\frac{1}{1+M} \sum_{i=0}^{M}\left[\sum_{n=0}^{m} \Delta_{n}^{R, \zeta}(i k \Delta x)\right]^{2},
\end{aligned}
$$

where $\Delta_{n}^{L, \phi}, \Delta_{n}^{R, \phi}, \Delta_{n}^{L, \zeta}$, and $\Delta_{n}^{R, \zeta}$ are given in Appendix, $M$ is the number of the discrete points, and $\Delta x=\pi / M$. In this paper, we choose $M=10$, and then the optimal convergencecontrol parameter $c_{0}$ is obtained by the minimum of $\varepsilon_{m}^{T}$.

\section{Results and Discussion}

To verify the convergence of our approximate analytic solutions to the nonlinear hydroelasticity problem considered here by means of the HAM, we employ the dimensional case of $\omega=2 \mathrm{~m}^{-1}, d=0.01 \mathrm{~m}, \rho_{\mathrm{e}}=0.9 \times 10^{3} \mathrm{kgm}^{-3}$, $\nu=0.33, E=10^{9} \mathrm{~Pa}, \rho=10^{3} \mathrm{kgm}^{-3}, t=2 \pi \mathrm{s}$, and $a_{0,1}=0.1 \mathrm{~m}$ and take these data hereinafter for calculation unless otherwise stated. The total residual square error $\varepsilon_{m}^{T}$ at different orders of approximation versus the convergencecontrol parameter $c_{0}$ is shown in Figure 2. It indicates that as the order of approximation $m$ increases gradually, the total residual square error $\varepsilon_{m}^{T}$ decreases in the interval $-0.5 \leq$

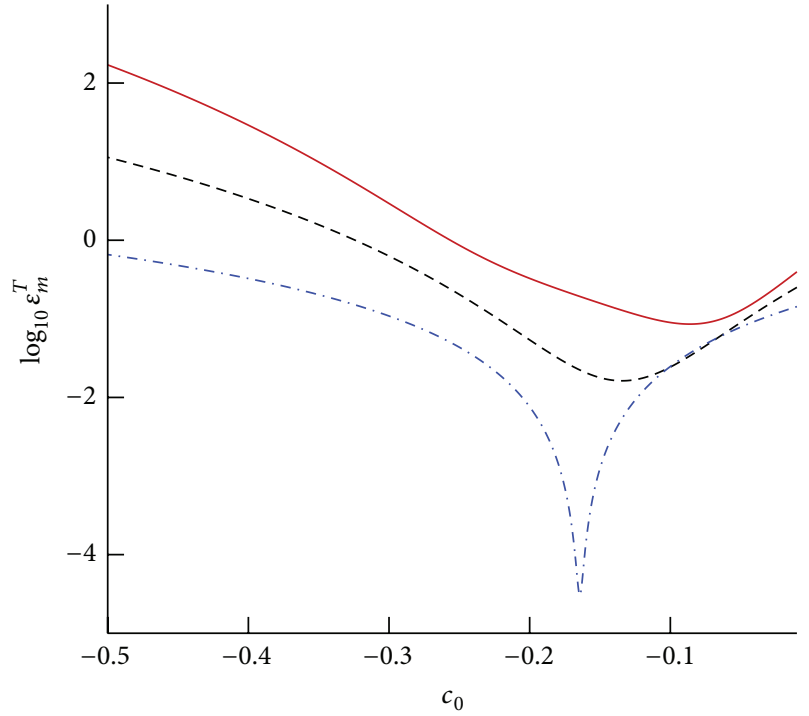

FIGURE 2: Residual squares of $\log _{10} \varepsilon_{m}^{T}$ versus $c_{0}$. Solid line: 1 st-order approximation; long dashed line: 2 nd-order approximation; dashdotted line: 3rd-order approximation.

TABLE 1: The total residual square error $\varepsilon_{m}^{T}$ for different approximation order $m$ with $c_{0}=-0.19$.

\begin{tabular}{lc}
\hline$m$ & $\varepsilon_{m}^{T}$ \\
\hline 1 & $4.11 \times 10^{-1}$ \\
3 & $3.94 \times 10^{-3}$ \\
5 & $2.83 \times 10^{-4}$ \\
7 & $3.16 \times 10^{-6}$ \\
\hline
\end{tabular}

$x \leq 0$, and the optimal value of $c_{0}$ is close to -0.19 . Further, when the optimal convergence-control parameter $c_{0}=-0.19$, the square error can decrease quickly to $2.83 \times 10^{-4}$ at the 5th-order approximation, as listed in Table 1 . These results indicate the validity and convergence of the corresponding HAM-based analytic solutions for velocity potential $\phi(x, z, t)$ and the water deflection $\zeta(x, t)$ governed by $(2)-(4),(6),(9)$, (10), and (11). It should be emphasized that the optimal $c_{0}$ in zeroth-order deformation equations such as (14) and (15) and (18) can help us to obtain relatively few terms of approximate solutions which have sufficiently small residual error.

Here we only change one physical parameter in the above case to exclude the effects of other ones on the nonlinear hydroelastic interaction between the incident progressive waves and the elastic plate. Firstly, we compare the HAMbased nonlinear solutions of hydroelastic response of a semiinfinite elastic plate floating on a fluid due to incident progressive waves with corresponding linear results also obtained by HAM. As shown in Figure 3, it can be seen clearly that our nonlinear homotopy-series approximation of the surface elevation $\zeta$ varies more greatly than the linear approximation, which further indicates that hydroelastic response of the floating plate will be underestimated if only linear theory is considered. In Figures 4-6, we give the incident progressive wave profiles and hydroelastic deflections of the plate for 


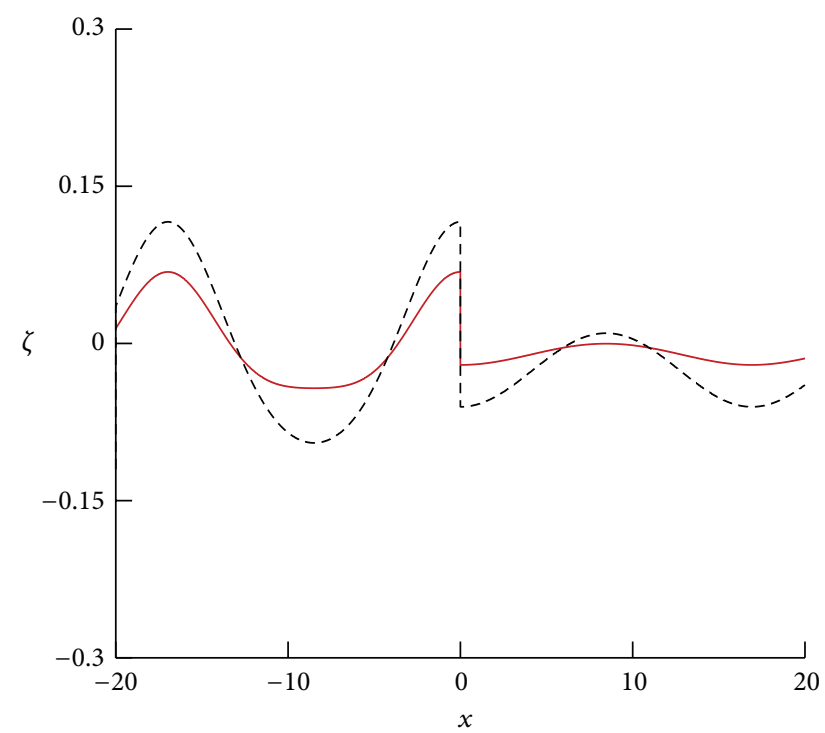

FIGURE 3: Comparison of my nonlinear wave profiles with those of corresponding linear theory. Solid line: linear solution; dashed line: nonlinear solution.

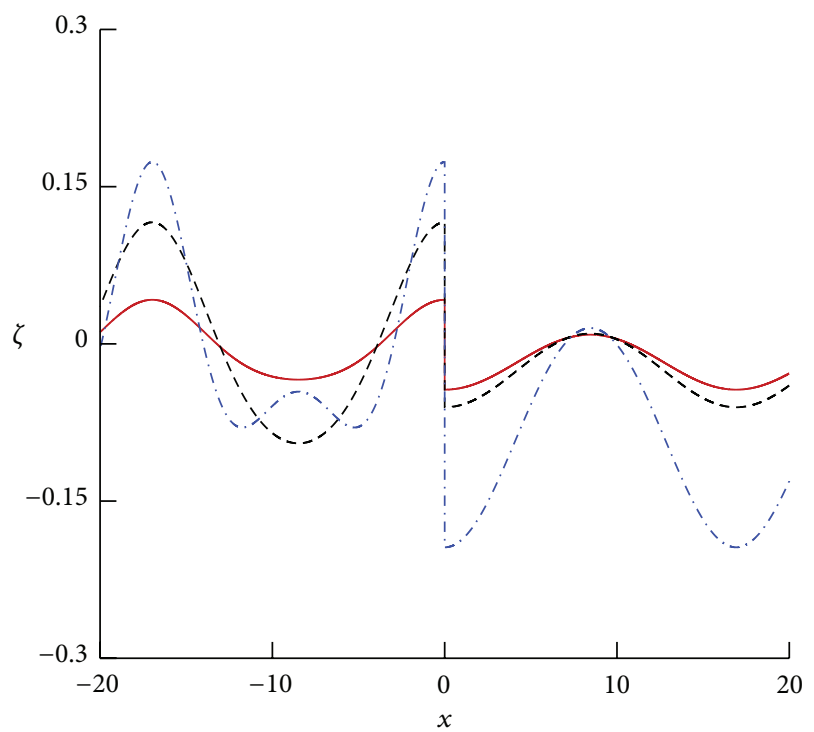

FIGURE 4: Variation of incident progressive waves and hydroelastic deflection of the plate versus $x$ for different amplitudes $a_{0,1}$ of initial incident wave. Solid line: $a_{0,1}=0.05 \mathrm{~m}$; dashed line: $a_{0,1}=0.1 \mathrm{~m}$; dash-dotted line: $a_{0,1}=0.2 \mathrm{~m}$.

different physical parameters. From the comparison between the open water and the plate-covered regions, it is easy to find that hydroelastic deflections of the plate are much smaller than the incident progressive wave profiles. This is due to the fact that, for the existence of a floating elastic plate, most of the wave energy is concentrated near the free surface. In other words, a large proportion of the wave energy is reflected back by the plate to the open water region and a small amount of wave energy is transmitted to the plate-covered region.

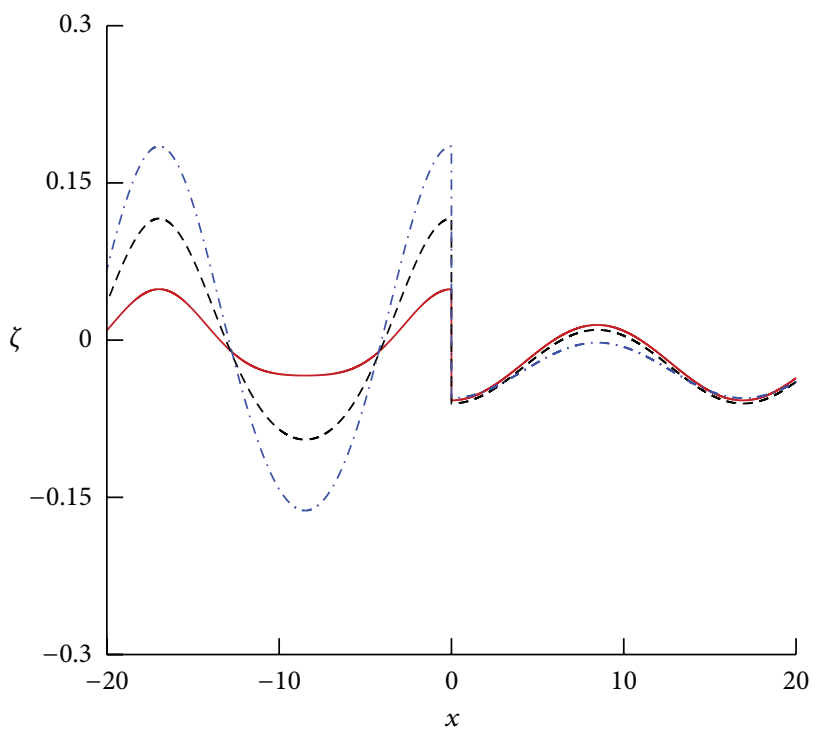

FIGURE 5: Variation of incident progressive waves and hydroelastic deflection of the plate versus $x$ for different values of Young's modulus $E$ of the plate. Solid line: $E=10^{8} \mathrm{~Pa}$; dashed line: $E=$ $10^{9} \mathrm{~Pa}$; dash-dotted line: $E=10^{10} \mathrm{~Pa}$.

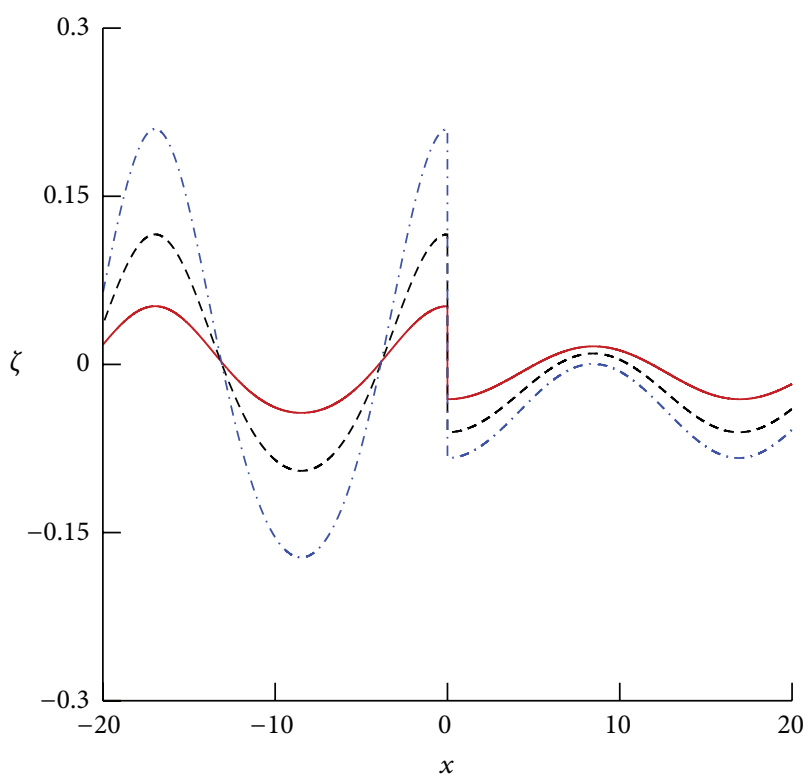

FIgURE 6: Variation of incident progressive waves and hydroelastic deflection of the plate versus $x$ for different plate thicknesses $d$. Solid line: $d=0.003 \mathrm{~m}$; dashed line: $d=0.01 \mathrm{~m}$; dash-dotted line: $d=$ $0.015 \mathrm{~m}$.

Practically speaking, in Figure 4 we give hydroelastic deflections of the plate and the incident wave profile by increasing incident wave amplitude $a_{0,1}$ from 0.05 to 0.5 . It is easy to find that the amplitudes of the plate defection increase with the increment of the amplitude $a_{0,1}$. The effect of Young's modulus of the plate is shown in Figure 5. And we can see that the incident progressive wave elevation in the open region increases while the hydroelastic deflection of 


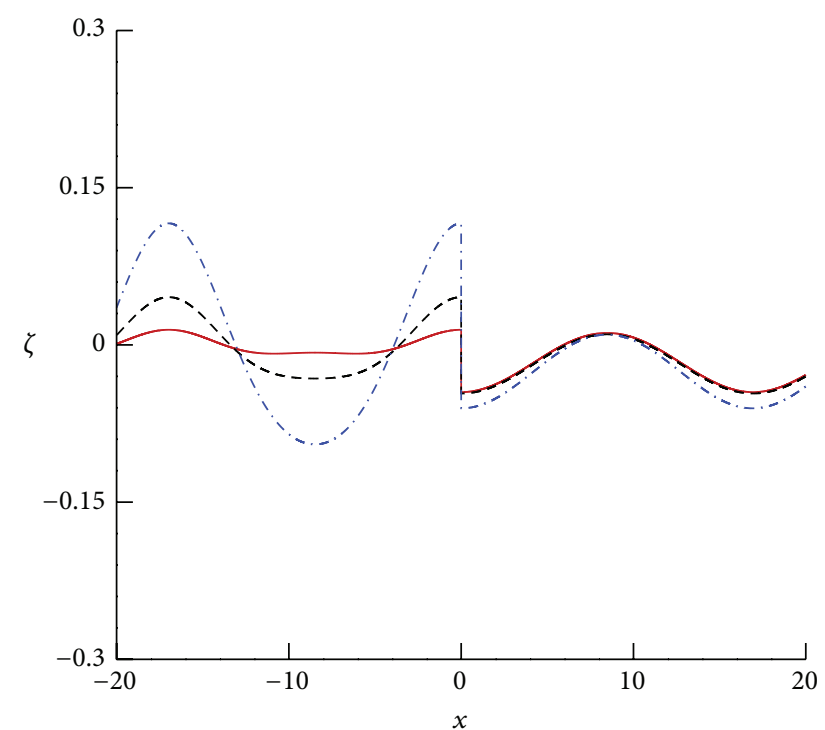

FIGURE 7: Variation of incident progressive waves and hydroelastic deflection of the plate versus $x$ for different plate densities $\rho_{\mathrm{e}}$. Solid line: $\rho_{\mathrm{e}}=0.1 \times 10^{3} \mathrm{kgm}^{-3}$; dashed line: $\rho_{\mathrm{e}}=0.5 \times 10^{3} \mathrm{kgm}^{-3}$; dashdotted line: $\rho_{\mathrm{e}}=0.9 \times 10^{3} \mathrm{kgm}^{-3}$.

the plate decreases as Young's modulus increases. This means the elastic plate becomes stiffer as Young's modulus increases, and then most of the incident progressive waves which concentrate near the interface between the plate-covered region and the open water region are reflected back, while less wave energy is transmitted below the plate. Figure 6 shows the differences of the wave deflections in the open water region and the plate-covered region for the plate thicknesses $d=0.003 \mathrm{~m}, d=0.01 \mathrm{~m}$, and $d=0.015 \mathrm{~m}$. It is found that a large thickness $d$ decreases the plate deflection while increasing the interfacial wave elevation. And as shown in Figure 7 , the plate density $\rho_{\mathrm{e}}$ has analogous influence on the incident progressive wave elevation in the open region and the hydroelastic deflection of the plate in the plate-covered region.

\section{Conclusions}

By the homotopy analysis method, convergent series solutions are derived for the free wave surface elevation and the deflection of a semi-infinite elastic plate floating on a fluid of infinite depth, respectively, to analytically study the nonlinear hydroelastic response of the plate with a free edge due to incident progressive waves. For the whole flow domain mathematically including an open water region and a platecovered region, we obtain solutions with fairly low residual error after computing only a small number of terms by introducing a convergence-control parameter. Further, in the plate-covered region, it is noted that all of two nonlinear operators $\mathcal{N}_{1}^{L}$ and $\mathcal{N}_{2}^{L}$ contain the linear operators for both $\zeta(x, t)$ and $\phi(x, z, t)$ mathematically. Fortunately, under the guarantee of the convergence of homotopy-series solutions, we may choose the auxiliary linear operator $\mathscr{L}_{1}^{L}$ containing the derivatives of $\phi(x, z, t)$ only and the auxiliary linear operator $\mathscr{L}_{2}^{L}$ containing the derivatives of $\zeta(x, t)$ only, which greatly simplify the calculation of nonlinear hydroelastic interaction. In addition, the relation of series solutions for these two regions is obtained by matching the velocity and pressure at boundary (11). Numerical results show that all our series solutions are highly convergent and accurate.

The influences of several physical parameters on the hydroelastic responses of the plate are considered in detail. The amplitude of incident wave, Young's modulus $E$, the thickness $d$, and density $\rho_{\mathrm{e}}$ of the floating elastic plate have effects on the free wave surface elevation and the plate deflection. As the amplitude of the incident wave increases, the plate deflection in the plate-covered region also becomes larger. With the increase of Young's modulus $E$ of the plate, the semi-infinite elastic plate deflection steps down, while the amplitude of free surface water elevation in the open water region becomes larger correspondingly. It is also found that the larger value of the thickness $d$ or density $\rho_{\mathrm{e}}$ of the plate can sharpen the crest and flatten the trough of the water elevation for the open water region significantly, and in the plate-covered region the plate deflection decreases. All of those results indicate that when incident progressive waves in the open water region interact on a stiffer or thicker plate, more wave energy is reflected into the open water region, while less wave energy is transmitted into the plate-covered region.

\section{Appendix}

Detailed Derivations of $\Delta_{m-1}^{L, \phi}, S_{m-1}^{L}, \bar{S}_{m}^{L}$, and $\Delta_{m-1}^{L, \zeta}$ in (40) and (41) for the Open Water

Region and $\Delta_{m-1}^{R, \phi}, S_{m-1}^{R}, \bar{S}_{m}^{R}$, and $\Delta_{m-1}^{R, \zeta}$ in (42) and (43) for the Plate-Covered Region

Let

$$
\eta^{n}=\left(\sum_{i=1}^{+\infty} \zeta_{i} q^{i}\right)^{n}=\sum_{i=n}^{+\infty} \mu_{n, i} q^{i}
$$

We have a Maclaurin series for any $z$ :

$$
\phi_{m}(x, z, t)=\left.\sum_{n=0}^{+\infty} \frac{1}{n !} \frac{\partial^{n} \phi_{m}}{\partial z^{n}}\right|_{z=0} z^{n} .
$$

For $z=\eta(x, t ; q)$, it follows from (A.1) and (A.2) that

$$
\begin{aligned}
\phi_{m}(x, t, \eta) & =\sum_{n=0}^{+\infty}\left(\left.\frac{1}{n !} \frac{\partial^{n} \phi_{m}}{\partial z^{n}}\right|_{z=0}\right)\left(\sum_{i=n}^{+\infty} \mu_{n, i} q^{i}\right) \\
& =\sum_{i=0}^{+\infty} \psi_{m, i} q^{i},
\end{aligned}
$$

where

$$
\psi_{m, i}=\sum_{n=0}^{i}\left(\left.\frac{1}{n !} \frac{\partial^{n} \phi_{m}}{\partial z^{n}}\right|_{z=0}\right) \mu_{n, i}
$$


Thus we have, for $z=\eta(x, t ; q)$,

$$
\begin{aligned}
\Phi(x, t, \eta ; q) & =\sum_{m=0}^{+\infty} \phi_{m}(x, t, \eta) q^{m}=\sum_{m=0}^{+\infty}\left(\sum_{n=0}^{+\infty} \psi_{m, i} q^{i}\right) q^{m} \\
& =\sum_{m=0}^{+\infty} \varphi_{m} q^{m}
\end{aligned}
$$

where

$$
\varphi_{m}=\sum_{i=0}^{m} \psi_{m-i, i}
$$

Substituting expansions (A.1) and (A.5) into the boundary conditions (14) and (15), then equating the like-power of $q$, we have two linear boundary conditions (40) and (41) for the open water region, respectively. Similarly, we can have two linear boundary conditions (42) and (43) for the platecovered region, respectively. And the explicit expressions for $\Delta_{m-1}^{L, \phi}, S_{m-1}^{L}, \bar{S}_{m}^{L}, \Delta_{m-1}^{L, \zeta}$ and $\Delta_{m-1}^{R, \phi}, S_{m-1}^{R}, \bar{S}_{m}^{R}, \Delta_{m-1}^{R, \zeta}$ in these conditions are given by

$$
\begin{aligned}
\Delta_{m-1}^{L, \phi}= & \frac{\partial^{2} \varphi_{m-1}}{\partial t^{2}}+g \bar{\varphi}_{m-1} \\
& +\sum_{n=0}^{m-1}\left(\frac{\partial \varphi_{n}}{\partial x} \frac{\partial^{2} \varphi_{m-1-n}}{\partial t \partial x}+\bar{\varphi}_{n} \frac{\partial \bar{\varphi}_{m-1-n}}{\partial t}\right) \\
& -g \sum_{n=0}^{m-1} \frac{\partial \varphi_{n}}{\partial x} \frac{\partial \zeta_{m-1-n}}{\partial x}, \\
\Delta_{m-1}^{L, \zeta}= & \frac{\partial \varphi_{m-1}}{\partial t}+\frac{1}{2} \sum_{n=0}^{m-1}\left(\frac{\partial \varphi_{n}}{\partial x} \frac{\partial \varphi_{m-1-n}}{\partial x}+\bar{\varphi}_{n} \bar{\varphi}_{m-1-n}\right) \\
+ & g \zeta_{m-1}, \\
\Delta_{m-1}^{R, \phi}= & \frac{\partial^{2} \varphi_{m-1}}{\partial t^{2}}+g \bar{\varphi}_{m-1} \\
+ & \sum_{n=0}^{m-1}\left(\frac{\partial \varphi_{n}}{\partial x} \frac{\partial^{2} \varphi_{m-1-n}}{\partial t \partial x}+\bar{\varphi}_{n} \frac{\partial \bar{\varphi}_{m-1-n}}{\partial t}\right) \\
+ & \frac{1}{\rho}\left[D \frac{\partial^{5} \zeta_{m-1}}{\partial t \partial x^{4}}+m \frac{\partial^{3} \zeta_{m-1}}{\partial t^{3}}\right]-g \sum_{n=0}^{m-1} \frac{\partial \varphi_{n}}{\partial x} \frac{\partial \zeta_{m-1-n}}{\partial x} \\
\Delta_{m-1}^{R, \zeta}= & \frac{\partial \varphi_{m-1}}{\partial t}+\frac{1}{2} \sum_{n=0}^{m-1}\left(\frac{\partial \varphi_{n}}{\partial x} \frac{\partial \varphi_{m-1-n}}{\partial x}+\bar{\varphi}_{n} \bar{\varphi}_{m-1-n}\right)
\end{aligned}
$$

$$
\begin{gathered}
\Delta_{0}^{R, \zeta}=\frac{\partial \varphi_{0}}{\partial t}+\frac{1}{2}\left[\left(\frac{\partial \varphi_{0}}{\partial x}\right)^{2}+\bar{\varphi}_{0}^{2}\right]+\frac{1}{\rho} m_{\mathrm{e}}, \\
S_{m-1}^{L}=S_{m-1}^{R}=\sum_{i=0}^{m-2}\left(\frac{\partial^{2} \psi_{m-1-i, i}}{\partial t^{2}}+g \gamma_{m-1-i, i}\right), \\
\bar{S}_{m}^{L}=\bar{S}_{m}^{R}=\sum_{i=1}^{m-1}\left(\frac{\partial^{2} \psi_{m-i, i}}{\partial t^{2}}+g \gamma_{m-i, i}\right),
\end{gathered}
$$

where

$$
\begin{gathered}
\bar{\varphi}_{m-1}=\sum_{i=0}^{m-1} \gamma_{m-1-i, i}, \\
\gamma_{m-i, i}=\sum_{n=0}^{i} \frac{1}{n !}\left(\left.\frac{\partial^{n+1} \phi_{m-i}}{\partial z^{n+1}}\right|_{z=0}\right) \mu_{n, i} .
\end{gathered}
$$

\section{Conflict of Interests}

There is no conflict of interests in this paper. The author obtained the approximate analytical solutions of the PDEs considered here by means of computer algebra system like Mathematica.

\section{Acknowledgments}

This research was sponsored by the Natural Science Foundation of Shanghai under Grant no. 14ZR1416200, the National Natural Science Foundation of China under Grant no. 11472166, and the Natural Science Foundation of Shandong Province of China under Grant no. ZR2013AL012.

\section{References}

[1] A. G. Greenhill, "Wave motion in hydrodynamics," The American Journal of Mathematics, vol. 9, no. 1, pp. 62-96, 1886.

[2] D. V. Evans and T. V. Davies, "Wave-ice interaction," Tech. Rep., DTIC Document, 1968.

[3] C. Fox and V. A. Squire, "On the oblique reflexion and transmission of ocean waves at shore fast sea ice," Philosophical Transactions of the Royal Society of London. Series A: Physical and Engineering Sciences, vol. 347, no. 1682, pp. 185-218, 1994.

[4] C. Fox and V. A. Squire, "Reflection and transmission characteristics at the edge of shore fast sea ice," Journal of Geophysical Research: Oceans, vol. 95, no. C7, pp. 11629-11639, 1990.

[5] V. A. Squire, J. P. Dugan, P. Wadhams, P. J. Rottier, and A. K. Liu, "Of ocean waves and sea ice," Annual Review of Fluid Mechanics, vol. 27, no. 1, pp. 115-168, 1995.

[6] T. Sahoo, T. Yip, A. T. Chwang et al., "On the interaction of surface waves with a semi-infinite elastic plate," in Proceedings of the 10th International Offshore and Polar Engineering Conference, International Society of Offshore and Polar Engineers, 2000.

[7] B. Teng, L. Cheng, S. X. Liu, and F. J. Li, "Modified eigenfunction expansion methods for interaction of water waves with a semiinfinite elastic plate," Applied Ocean Research, vol. 23, no. 6, pp. 357-368, 2001. 
[8] F. Xu and D. Q. Lu, "An optimization of eigenfunction expansion method for the interaction of water waves with an elastic plate," Journal of Hydrodynamics, vol. 21, no. 4, pp. 526-530, 2009.

[9] Q. Lin and D. Q. Lu, "Hydroelastic interaction between obliquely incident waves and a semi-infinite elastic plate on a two-layer fluid," Applied Ocean Research, vol. 43, pp. 71-79, 2013.

[10] L. K. Forbes, "Surface waves of large amplitude beneath an elastic sheet. Part 1. High-order series solution," Journal of Fluid Mechanics, vol. 169, pp. 409-428, 1986.

[11] L. K. Forbes, "Surface waves of large amplitude beneath an elastic sheet. Part 2. Galerkin solution," Journal of Fluid Mechanics, vol. 188, pp. 491-508, 1988.

[12] J.-M. Vanden-Broeck and E. I. Părău, "Two-dimensional generalized solitary waves and periodic waves under an ice sheet," Philosophical Transactions of the Royal Society A: Mathematical, Physical and Engineering Sciences, vol. 369, no. 1947, pp. 29572972, 2011.

[13] P. A. Milewski, J.-M. Vanden-Broeck, and Z. Wang, "Hydroelastic solitary waves in deep water," Journal of Fluid Mechanics, vol. 679, pp. 628-640, 2011.

[14] S. Liao, The proposed homotopy analysis technique for the solution of nonlinear problems [Ph.D. thesis], Shanghai Jiao Tong University, Shanghai, China, 1992.

[15] S.-J. Liao, "On the homotopy multiple-variable method and its applications in the interactions of nonlinear gravity waves," Communications in Nonlinear Science and Numerical Simulation, vol. 16, no. 3, pp. 1274-1303, 2011.

[16] D. Xu, Z. Lin, S. Liao, and M. Stiassnie, "On the steady-state fully resonant progressive waves in water of finite depth," Journal of Fluid Mechanics, vol. 710, pp. 379-418, 2012.

[17] P. Wang and D. Lu, "Analytic approximation to nonlinear hydroelastic waves traveling in a thin elastic plate floating on a fluid," Science China Physics, Mechanics and Astronomy, vol. 56, no. 11, pp. 2170-2177, 2013.

[18] S. Liao, Beyond Perturbation: Introduction to the Homotopy Analysis Method, vol. 2, CRC Press, Boca Raton, Fla, USA, 2004.

[19] S. Liao, Homotopy Analysis Method in Nonlinear Differential Equations, 2012. 


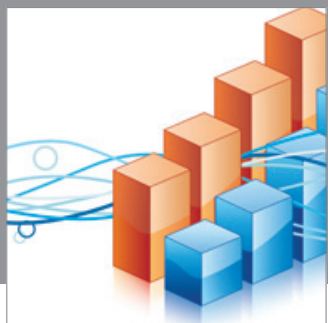

Advances in

Operations Research

mansans

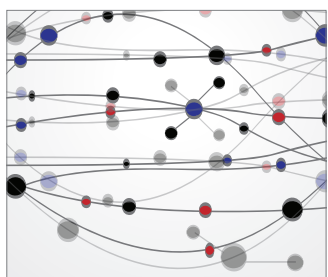

The Scientific World Journal
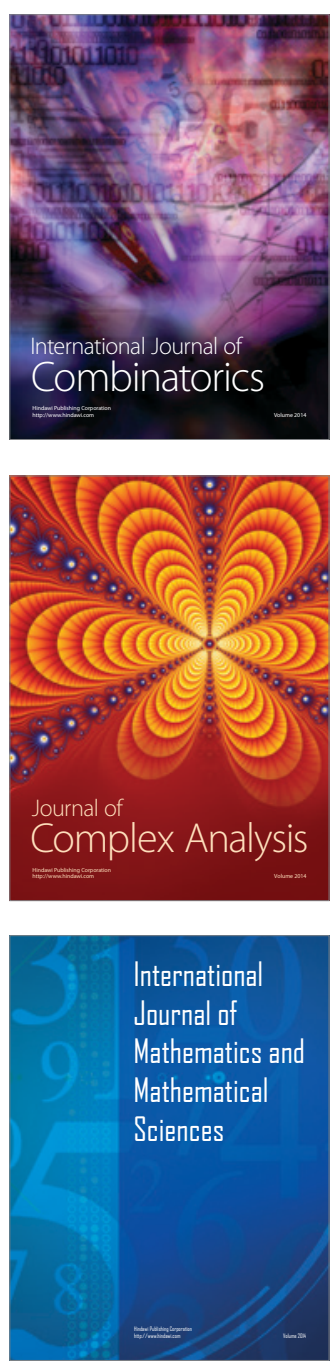
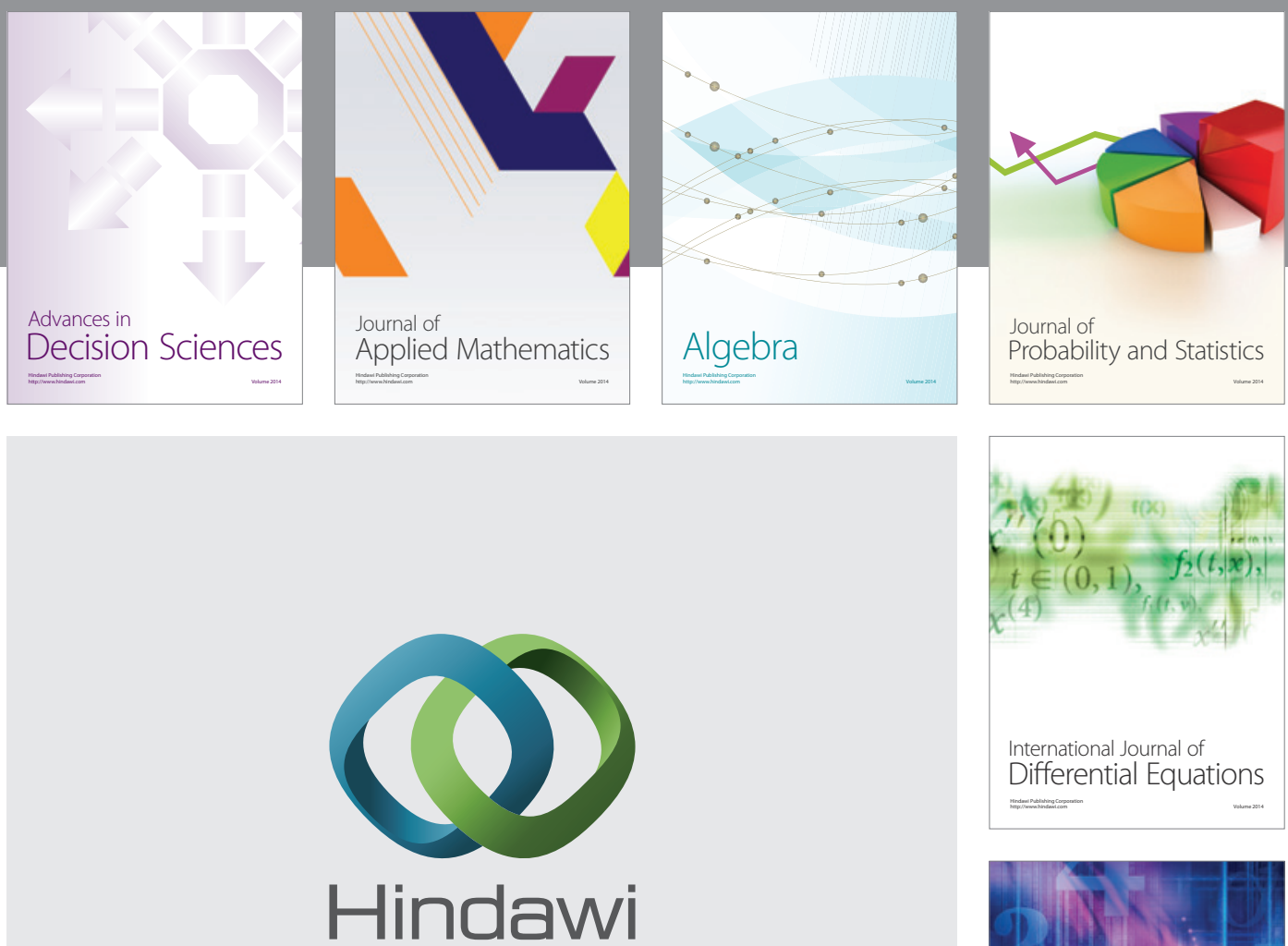

Submit your manuscripts at http://www.hindawi.com
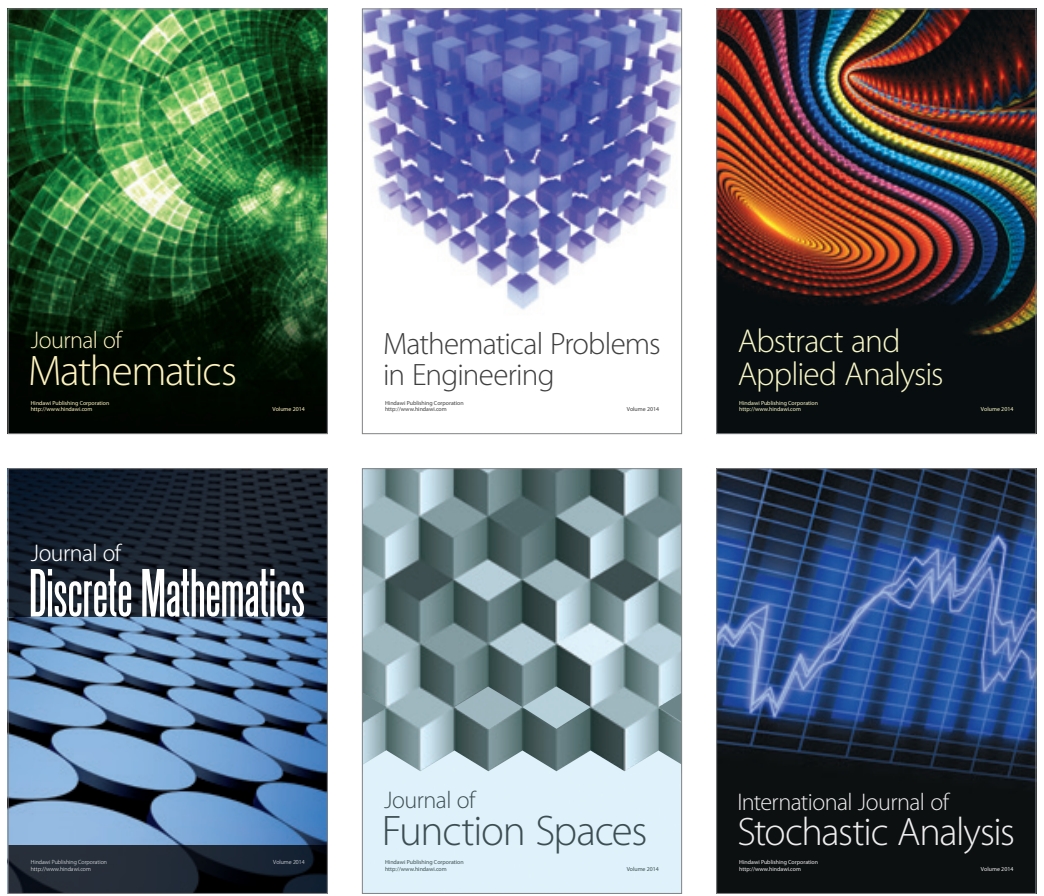

Journal of

Function Spaces

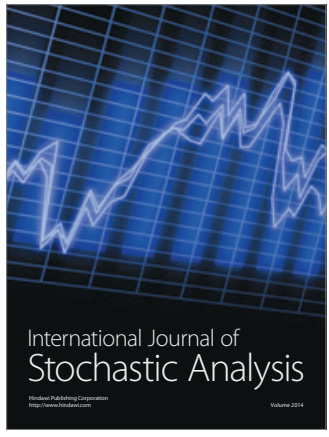

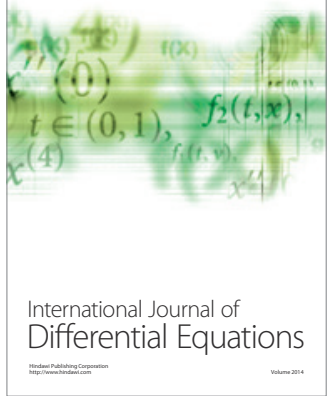
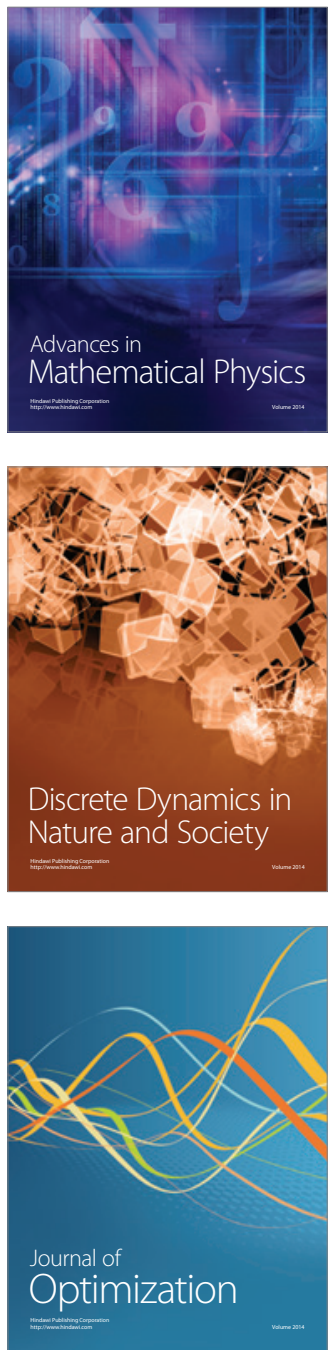